\title{
Dimensioning 1,2,3
}

\author{
Chiara Passa \\ Rome, Italy \\ chiarapassa@gmail.com
}

\section{INTRODUCTION}

"Dimensioning 1,2,3" are three virtual reality animations part of 'Live Architectures', a series of digital artworks I created over time in a multifaceted production developed to shake-up the static concept of design and exploring architecture as interface.

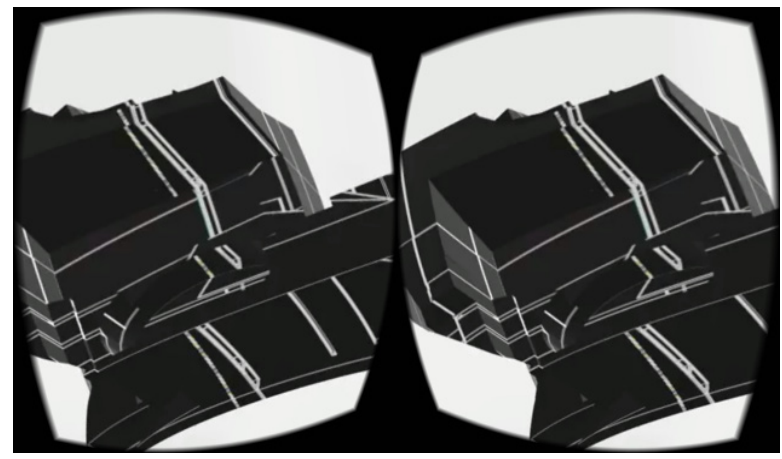

Figure 1: Still from Dimensioning $n$ 1, VR animation.

Live Architectures series analyse changes in liquid space through a variety of techniques, technologies and devices, often using augmented reality and virtual reality as mediums. Animations, video, installations, net art, interactive projects and videomapping, all become instruments of research targeted at the different ways space is configured and how it is generated from electronic language, from the interaction with humans to the way they blend and melt together at a given point.

\section{DIMENSIONING SERIES}

I have designed the virtual reality series "Dimensioning" to create a multi-dimensional extension of place, allowing visitors travel through the space and into the moving diagrams. Fragile lines form complex architectural shapes create the impression of walking through a geometric equation. The complex shape becomes a window into a virtual world, where the moving diagrams take over and a new architectural dimension takes shape.

The Live Architectures I created, act as if they were alive and vibrant, just moving throughout the $3 \mathrm{D}$ viewer. So, a performance idea is the base of those artworks, where the spectators perceive and receive a place moving all around, beyond its functionality. Therefore, the term "Super-place" । coined on 1999 for Live Architectures series (1999ongoing) includes also the VR series "Dimensioning 1,2,3", as dynamic places which project the spectator into a digital reality. Exactly the contrary happens in the 'no-where' in which static presences have only the function to receive temporarily.

It is just the notion of space that "Dimensioning VR" wants to probe, by searching new possibilities and 'dimensions', that the digital world, not so much separated from that real-one offer us everyday.

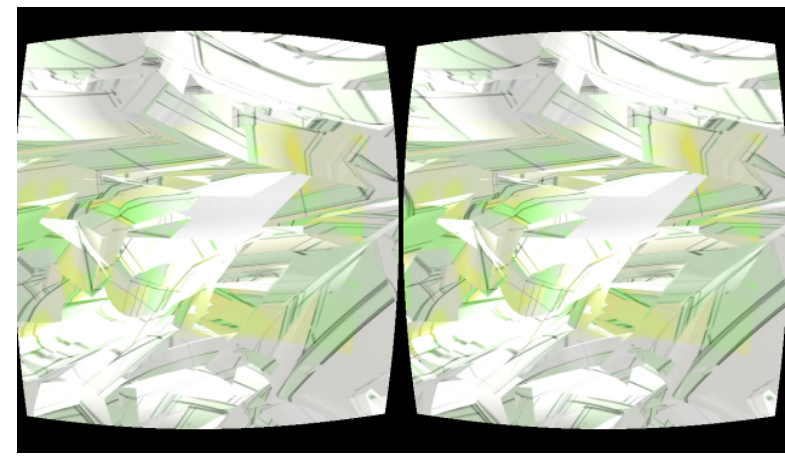

Figure 2: Still from Dimensioning $n$ 2, VR animation.

\subsection{Virtual Reality and the viewer}

The unreal figure becomes design, structure, architecture and mediated reality. If the space is the extension in all the directions by our intuitions of the real world in which material bodies are placed, my artwork wants to expand these possibilities of perception. It is through experience that Dimensioning series addresses the paradox of the modern space-time condition, diluted between 
physical and liquid space. The spectator becomes blind and ultra-seeing at the same time, forced to immerse herself/himself in the shifts generated by his perception of the variable geometric language. introduces a plus valorem: it gives the opportunity to enjoy digital artwork privately - albeit surrounded by a crowd - via the 3D visor, endorsing a kind of intimate dimension between the person viewing and the work of art.
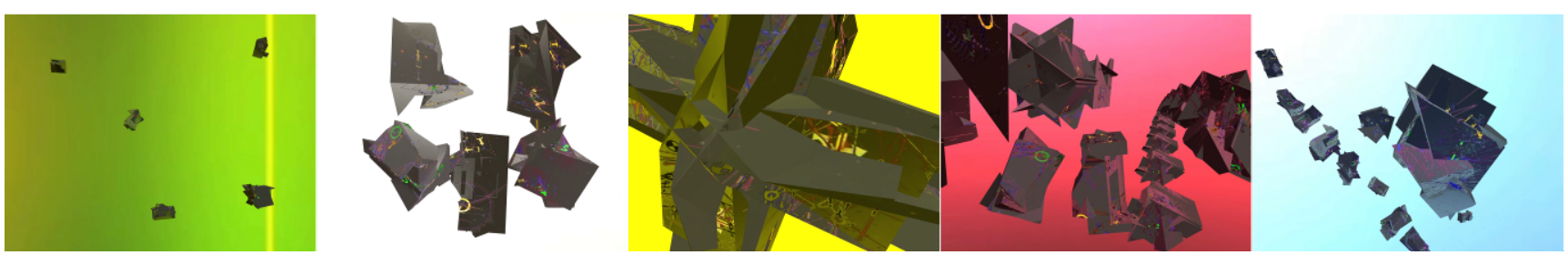

Figure 2: Stills from Dimensioning n 3, VR Animation.

\section{AUTHOR REFERENCES}

Chiara Passa, visual artist based in Rome. I received an M.F.A. from the Fine Arts Academy of Rome and a Master in new audio-visual mediums from the Faculty of Modern Literature. My artwork explore architecture as interface combining different media as: Internet-art projects, animations, interactive video-installations, digital art in public space as site-specific artworks, video-mapping and video-sculptures. I also develop art-applications (AR and VR) and widgets for mobile platforms. Since1997 my artworks were internationally exhibited from Festivals \& institutions, as for example: "Dimensioning" at Furtherfield gallery, (London, UK), ISEA Vancouver Art Gallery (Canada), Vortexdome (L.A. USA), Media Art Histories Conference (Riga, Latvia), Electrofringe (New Castle, Australia), FILE | Electronic Language International Festival (Sao Paulo, Brazil), MAK Museum of contemporary art (Vienna, Austria), CCCB - Centro de Cultura Contemporanea Barcelona (Barcelona, Spain). Further details on
In fact, by means of virtual reality space, the spectator is absorbed into an illusory dimension which redraws the entire surroundings, eliminating corporal limits and projecting the vision beyond imagination, thus challenging the notion of architecture and exploring it as interface. my artistic research, full CV/statement and shows can be found at:

http://www.chiarapassa.it/Artisticprofile.html http://www.chiarapassa.it/SelectedExhibitions.html and on Wikipedia at:

https://en.wikipedia.org/wiki/Chiara Passa

\section{Conference and shows where "Dimensioning" VR series was exhibited:}

"Dimensioning n 1" was exhibited at Furtherfield gallery, London, April 2016.

"Dimensioning n 1,2,3" at CAC.05 - Computer Art Congress and exhibition: 'Digital Art: Archiving and Questioning Immateriality', Paris, October 2016.

"Dimensioning $\mathrm{n}$ 1" was exhibited at Besides the Screen festival, Coventry UK \& Sao Paulo, 2016.

"Dimensioning $\mathrm{n}$ 1" was exhibited at at Cairotronica, international electronic media art festival El Cairo, 2016. 